\title{
Effects and mechanisms of Eps8 on the biological behaviour of malignant tumours (Review)
}

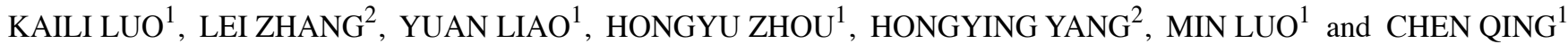 \\ ${ }^{1}$ School of Pharmaceutical Sciences and Yunnan Key Laboratory of Pharmacology for Natural Products, \\ Kunming Medical University, Kunming, Yunnan 650500; ${ }^{2}$ Department of Gynecology, Yunnan Tumor Hospital \\ and The Third Affiliated Hospital of Kunming Medical University; Kunming, Yunnan 650118, P.R. China
}

Received August 29, 2020; Accepted December 9, 2020

DOI: $10.3892 /$ or.2021.7927

\begin{abstract}
Epidermal growth factor receptor pathway substrate 8 (Eps8) was initially identified as the substrate for the kinase activity of EGFR, improving the responsiveness of EGF, which is involved in cell mitosis, differentiation and other physiological functions. Numerous studies over the last decade have demonstrated that Eps8 is overexpressed in most ubiquitous malignant tumours and subsequently binds with its receptor to activate multiple signalling pathways. Eps8 not only participates in the regulation of malignant phenotypes, such as tumour proliferation, invasion, metastasis and drug resistance, but is also related to the clinicopathological characteristics and prognosis of patients. Therefore, Eps 8 is a potential tumour diagnosis and prognostic biomarker and even a therapeutic target. This review aimed to describe the structural characteristics, role and related molecular mechanism of Eps8 in malignant tumours. In addition, the prospect of Eps8 as a target for cancer therapy is examined.
\end{abstract}

\section{Contents}

1. Introduction

2. Structure and function of Eps8

3. Abnormal expression of Eps8 in malignant tumours and tumorigenesis

4. Role and molecular mechanism of Eps8 on solid tumours

5. Role and molecular mechanism of Eps8 in hematological tumours

6. Conclusion and future perspective

Correspondence to: Professor Chen Qing or Dr Min Luo, School of Pharmaceutical Sciences and Yunnan Key Laboratory of Pharmacology for Natural Products, Kunming Medical University, 1168 West Chunrong Road, Yuhua Avenue, Chenggong, Kunming, Yunnan 650500, P.R. China

E-mail: qingchenhhh@qq.com

E-mail: feiyue1998@163.com

Key words: epidermal growth factor receptor pathway substrate 8, malignant tumours, proliferation, migration, invasion

\section{Introduction}

Malignant tumours are uncontrolled cell proliferation diseases caused by oncogenes and ultimately lead to organ and body dysfunction (1). In recent decades, great progress has been made in the study of genes and signalling pathways in tumorigenesis. Eps8 was identified by Fazioli et al in NIH-3T3 murine fibroblasts via an approach that allows direct cloning of intracellular substrates for receptor tyrosine kinases (RTKs) that was designed to study the EGFR signalling pathway. Eps8 is mainly distributed in epithelial cells and fibroblasts as well as in some, but not all, haematopoietic cells located in the cytoplasm, nuclear membrane and around the cell membrane (2). As a tyrosine kinase receptor, Eps8 maps to human chromosome $12 \mathrm{p} 12.3$ and consists of 821 amino acids (3). In mammals, there are at least three genes highly homologous to Eps8, namely Eps8L1, Eps8L2, and Eps8L3, and they preserve the main structure of Eps8, thus defining a new gene family (4,5). Eps8s are expressed differently during development but are typically co-expressed in adults (4). Studies have indicated that, at the mRNA level, the expression pattern of Eps8 overlaps with that of Eps8L2, and their expression is relatively extensive; in contrast, Eps8L1 and Eps8L3 displays restricted expression in adult tissues (4). Notably, Eps8L1 and Eps8L2, which have the highest homology with the C-terminal effect region of Eps8, may compensate for Eps8 function in the whole organism $(4,5)$, and among them, Eps 8 is the only selectively upregulated subtype in the brain (6). There are two subtypes of proteins recognized by Eps8 antibodies: p97Eps8 and p68Eps8 (2). Both of these subtypes are certified as substrates for several RTKs (7). The gene encoding p97Eps8 is an oncogene whose $\mathrm{PH}$ domain is critical for ERK activation, cell localization, and cell transformation (7). Furthermore, Eps8 binds to actin in vivo and accumulates in PDGF-induced ruffles (6). In brief, Eps8 is an essential protein encoding the Ras and Rac signalling pathways and is effectively phosphorylated by a variety of tyrosine kinases (receptor and non-receptor types), resulting in actin remodelling (2).

Eps8 is highly conserved and is widely expressed during mouse development $(8,9)$. Studies mainly focused on humans have confirmed that Eps8 is markedly expressed in diverse types of solid tumours (10-23) and even haematological malignancies (24-27) but minimally expressed in normal 
tissues (Table I). The aberrant expression of Eps8 is related to numerous signalling pathways, which affect a series of biological processes by regulating various downstream cascades, such as EGFR transduction, actin dynamics, cell cycle regulation and cell proliferation, and eventually tumours tend to undergo malignant transformation (10-27). In addition, Eps8 is also a new pathogenic gene for autosomal recessive profound deafness that encodes the actin of cochlear hair cell stereocilia (28). In summary, Eps8 potentially represents a novel biomarker for cancer diagnosis and a promising candidate for cancer therapy.

\section{Structure and function of Eps8}

Structure of EPS8. The Eps8 amino acid sequence predicted by computer-aided analysis reveals a typical signal molecular structure. From the $\mathrm{N}$-terminus to the $\mathrm{C}$-terminus, there is a phosphate binding protein (PTB) region, proline-rich sequences and SH3 region, stereo alpha-pointed (SAM-PNT) domain (5) (Fig. 1). It should be noted that the research on the $\mathrm{SH} 3$ domain is relatively extensive, and we have a greater understanding of this domain.

The SH3 domain is a protein component identified in the study of Src. Proteins with this domain recognize peptides containing XPXXP via sequence similarity and bind these peptides (29). In particular, the SH3 domain of Eps8 exhibits a novel and unique binding preference, mainly binding to peptides containing PXXDY rather than canonical XPXXP, establishing specific interactions in the signal network. Notably, the SH3 domain of Eps8 can interact with a number of binding partners. When combined with shc (30), shb (31), RN-tre (32,33), E3B1 (Abi-1) (32,34), Dvl-1 (35) and IRSp53 (36), Rac-mediated actin remodelling is activated; however, when combined with RN-tre, Rab5-mediated EGFR internalization is inhibited (33).

Proline-rich sequences are well characterised for the adaptor protein IRSp53, which is linked to Rho family small GTPases and exists in fibroblasts and various cancer cell lines. The proline-rich sequence in the N-terminal region directly binds to IRSp53 to form a complex. This binding subsequently mediates the positive regulation of Rac activity by enhancing the formation of the Eps8/Abi-1/Sos-1 complex and coactivating Rac. Furthermore, the formation of the IRSp53/Eps8 complex at the leading edge of motor cells is closely related to cell movement and invasiveness $(36,37)$.

The N-terminus contains a functional PTB domain (4), which acts as a module for protein-protein interactions, connecting the catalytic domain of tyrosine kinase (38), and combining different peptides in a phosphorylation-dependent or phosphorylation-independent manner. The interaction involves different biological processes ranging from types of receptor signals to protein localization (39). Nevertheless, no binding partner for the PTB domain of Eps8 is yet known.

The SAM-PNT domain belongs to the subfamily of SAM domains and mediates the homo- and hetero-oligomerization of proteins and the interaction of specific proteins (40). Others aspects have not been discovered.

Physiological function of EPS8. A structure-function study concluded that Eps8 contains two functional regions. The first region discussed is the EGFR binding region, which is rarely studied. This region acts as a binding surface for the juxtamembrane region of EGFR and is connected with mitosis, but its mechanism remains unclear (41). It is hypothesized that this region may help the recruitment of Eps8 and Eps8-based complexes to EGFR, facilitating downstream signal propagation and mitotic stimulation (42).

The other region is defined as the C-terminus 'effector region'. First, it regulates Rac-specific catalytic GEF activity by binding SOS-1, which directly affects filamentous actin remodelling (32). Moreover, the localization of Eps8 cells is implied by mediating the interaction between Eps8 and F-actin in vivo. Consequently, phosphorylation may not only participate in the catalytic activation of the Eps8-Abi1-Sos1 signalling complex but also indicate Eps8 and Eps8-based complex localization, thereby mediating the actin-based movement process in the cells $(42,43)$. Additionally, Eps8 plays a pivotal role in membrane flow, the formation of pseudopods, the morphogenesis of microvilli, the function and length of static cilia, cell adhesion and motility (15).

\section{Abnormal expression of Eps8 in malignant tumours and tumorigenesis}

Eps8 is universally expressed in human tissues, especially in the gall bladder, fat, colon, small intestine, kidney, endometrium, placenta, ovary and bladder $(4,6)$. Numerous studies have demonstrated that Eps8 is unconventionally expressed in various tumour types, and high levels of Eps 8 promote tumour proliferation in breast cancer $(11,20)$, pancreatic cancer (13), colon cancer (14), pituitary tumour (16), oesophageal cancer (17), non-small cell lung cancer (NSCLC) (21), and glioblastoma (22). In addition, Eps8 can also improve the migration ability of cancer cells, including oral squamous cell carcinoma (OSCC) (15) and colon, breast and ovarian cancer $(12,18,20)$. Moreover, the aberrant expression of Eps8 alters the sensitivity of cervical cancer cells to anticancer drugs (14) and is closely related to the prognosis of OSCC and pancreatic adenocarcinoma (PDAC) $(19,23)$, thus affecting the quality of life of patients. Aside from common malignant solid tumours, recent research has revealed that Eps8 is also aberrantly expressed in malignant haematological tumours; similarly, it regulates the development of a series of tumours, including acute lymphoblastic leukaemia (ALL) (24), chronic myeloid leukaemia (CML) (25), acute myeloid leukaemia (AML) (26) and multiple myeloma (MM) (27). Overall, Eps8 can predict tumorigenesis and even tumour progression. Blocking Eps8 can inhibit tumour proliferation, metastasis, and drug resistance and improve the overall survival rate of tumour patients. Therefore, Eps8 is anticipated to become a detection index and a new therapeutic target for malignant tumours.

Hyperactivation of intracellular signalling pathways is a key driver of numerous cancers. EGF, which is composed of a single peptide, is a mitogen of fibroblasts, epithelial cells and endothelial cells $(44,45)$. EGF binds specific receptors on the cell surface, controls EGFR dimerization, and activates tyrosine kinase and receptor trans-autophosphorylation, triggering multiple downstream cascades that promote cell proliferation $(46,47)$. Potentiation of the proliferative 
Table I. Summary of Eps8 overexpression in human malignant tumours.

\begin{tabular}{lccc}
\hline Type of tumour & Year & $\begin{array}{c}\text { Relative overexpression } \\
\text { value of Eps8 (total no. of cases) }\end{array}$ & $($ Refs.) \\
\hline PTC & 2001 & $8(8)$ & $(10)$ \\
Breast cancer & 2002 & 2.77 -fold & $(11)$ \\
Colon cancer & 2007 & $47(76)$ & $(12)$ \\
PDAC & 2007 & 4 -fold & $(13)$ \\
Cervical cancer & 2008 & $45(75)$ & $(14)$ \\
OSCC & 2009 & $>5$-fold & $(16)$ \\
Pituitary tumour & 2009 & 5.9 -fold & $(17)$ \\
ESCC & 2010 & $35(65)$ & $(18)$ \\
Ovarian cancer & 2010 & $63.50 \%$ & $(19)$ \\
OSCC & 2012 & $186(205)$ & $(20)$ \\
Breast cancer & 2015 & $60 \%$ & $(24)$ \\
ALL & 2015 & High risk & $(25)$ \\
CML & 2018 & $50(91)$ & $(26)$ \\
AML & High & $(27)$ \\
MM & 2018 & High & $(21)$ \\
NSCLC & 2019 & High & $(22)$ \\
GBM & 2019 & Significantly higher & $(23)$ \\
PDAC & 2019 & $31(46)$ &
\end{tabular}

The third column of the table: $n$ (total number of tumors investigated), 'x-fold' or 'high risk' indicated that a specific value for the number of tumors with relative overexpression value of Eps8 was not provided. The binary risk classification of event prediction according to the threshold value was determined, and a value above this threshold indicated 'high risk' (24). Eps8, epidermal growth factor receptor pathway substrate 8; PTC, papillary thyroid carcinoma; PDAC, pancreatic ductal adenocarcinoma; OSCC, oral squamous cell carcinoma; ESCC, esophageal squamous cell carcinoma; ALL, acute lymphocytic leukemia; CML, chronic myeloid leukemia; AML, acute myeloid leukemia; MM, multiple myeloma; NSCLC, non-small cell lung cancer.

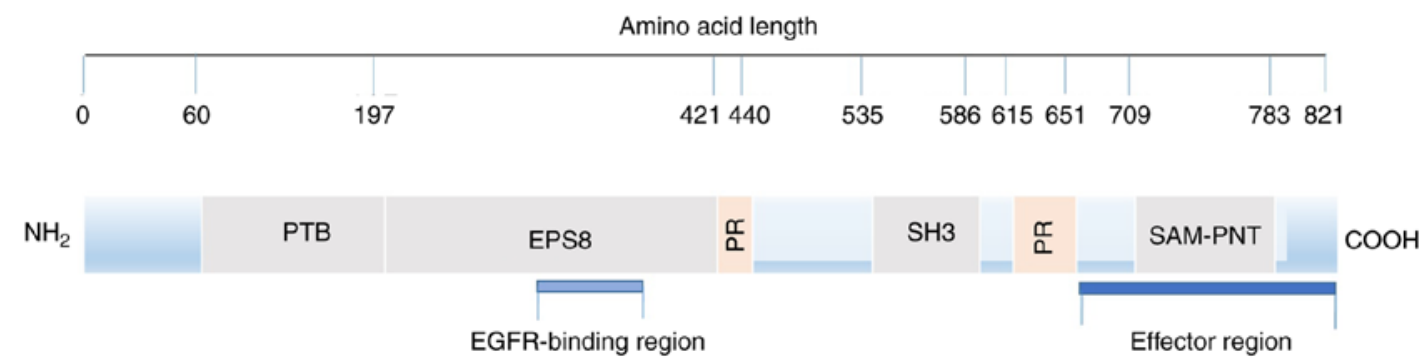

Figure 1. Eps8 structure diagram. Computer analysis predicts that human Eps8 is 821 amino acids long, from N-terminal to C-terminal including: A PTB region (amino acids: 60-197), an EGFR-binding region (amino acids: 298-362), two proline-rich sequences (amino acids: 421-440 and 615-651), an SH3 domain (amino acids: 535-586), a SAM-PNT domain (amino acids: 709-783), and an 'effector region' (amino acids: 648-821). The blue bar indicates the functional region of Eps8 (38). Eps8, epidermal growth factor receptor pathway substrate 8.

effects of EGF as a result of receptor overexpression and/or activation of its tyrosine kinase has long been considered to drive carcinogenesis (48). In general, Eps8 overexpression confers EGF-dependent mitotic signals $(2,30)$, which bind directly to the JXM region of EGFR and are phosphorylated. Several intracellular signalling pathways may become activated following EGFR stimulation, including inositol phosphoinositide 3-OH kinase (PI3K). Phospholipase C- $\gamma$ (PLC $\gamma$ ), activators of transcription (STATS) (49-51), ERK (52), JNK MAP kinases (53) and Src are also activated. In addition, the activation of c-jun $\mathrm{N}$ terminal kinase in a Rac-dependent manner is also mediated by EGFR (54).
Given that Eps8 mediates important biological processes, it is not surprising that it serves as an attractive molecular therapeutic target and even a prognostic marker.

\section{Role and molecular mechanism of Eps8 in solid tumours}

The aberrant expression of Eps8 in most tumours is involved in tumour progression; notably, its expression level may contribute to tumour proliferation, invasion and metastasis, drug resistance and prognosis. Importantly, the mechanisms involved are intricate (Fig. 2). Therefore, studying the biological significance of Eps8 in solid tumours and its molecular 


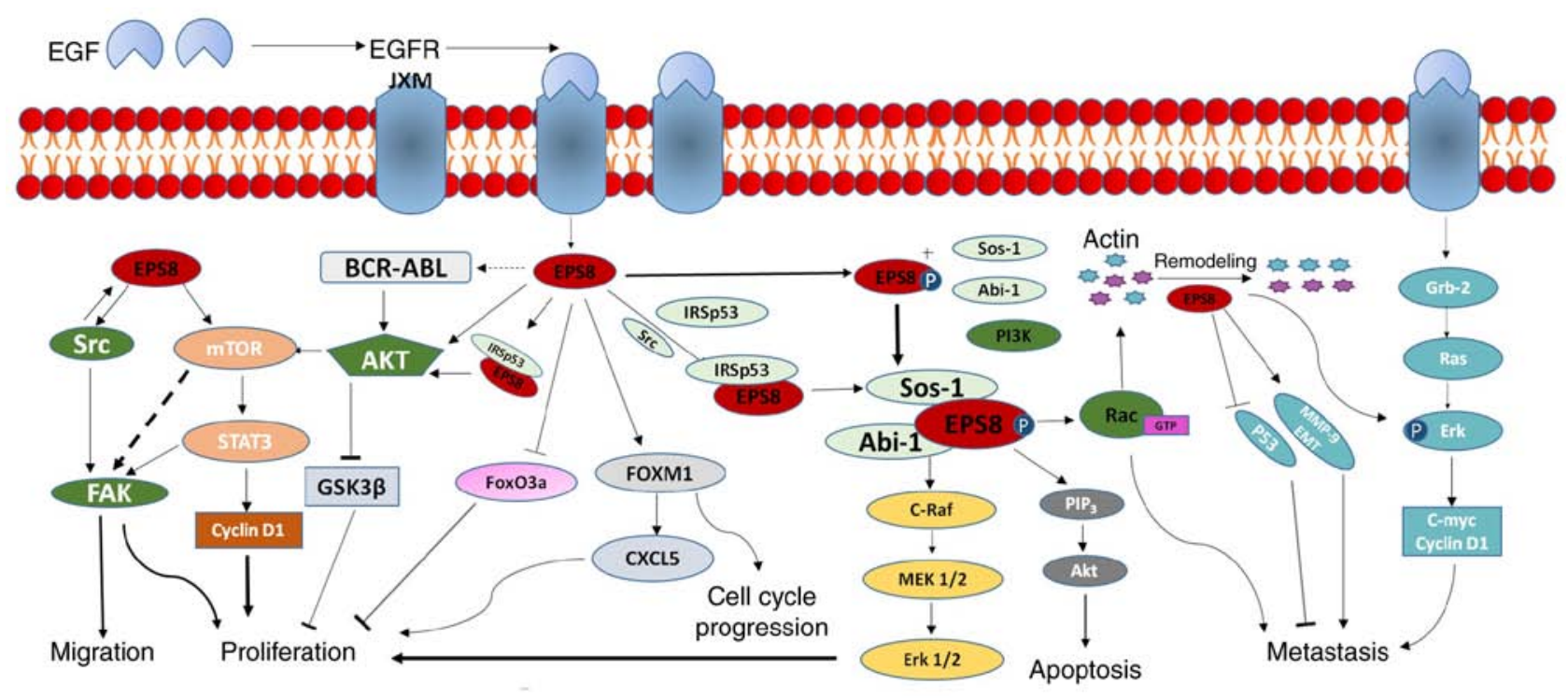

Figure 2. Role of Eps8 in the regulation of the malignant phenotype of a tumour. Eps8 enhances EGF-dependent mitotic signals with a complex mechanism. It binds to its receptor EGFR and activates multiple related signaling pathways, which are involved in the regulation of biological effects such as tumour proliferation, apoptosis, migration and metastasis. Eps8, epidermal growth factor receptor pathway substrate 8 .

mechanisms will facilitate further exploration of cancer therapy strategies targeting Eps8.

\section{Role and mechanisms of EPS8 in tumour proliferation}

Eps 8 regulates tumour proliferation. As early as the beginning of the 21st century, studies emphasized that Eps 8 is highly expressed in tumour cells (10-20) and can modulate proliferation $(11,13,14,16,17,20)$, which ignited the enthusiasm of researchers for the ability of Eps8 to regulate the malignant phenotype of tumours. In general, the pathways involved in regulating proliferation include the mTOR/STAT3/FAK pathway and PI3K/AKT pathway.

mTOR/STAT3/FAK signalling pathway and tumour proliferation. Eps8 determines the expression of downstream factors required for cell proliferation, and the expression level of Eps8 in colorectal cancer can reflect cell proliferation ability. The attenuation of Eps8 reduces FAK, an intracellular tyrosine kinase that exhibits prominent local adhesion $(55,56)$, and is involved in a variety of integrin-induced biological activities, including cell migration, growth and survival $(57,58)$. Further research revealed that this phenomenon is integrated through the mTOR/STAT3 pathway. Eps8 overexpression activates mTOR, a proprotein kinase that promotes protein synthesis, which subsequently triggers mTOR to induce FAK and cyclin D1 expression, leading to tumorigenesis and tumour proliferation (12). Similarly, Eps8 and FAK expression trends were detected in tumour specimens, especially in advanced patients (12). In addition, it has been reported that STAT3 is continuously activated in src-transformed cells (59), and the expression of dominant-negative STAT3 markedly abrogates src-induced transformation $(60,61)$. Eps 8 overexpression markedly increases src activity $(62,63)$, and src subsequently promotes tyrosine phosphorylation and Eps8 protein synthesis, stimulating FAK expression and activity (64). Collectively, Eps 8 and FAK are important in the process of tumour proliferation.
Growth factor and cell proliferation. Continuous proliferation signals in cancer cells may be the constitutive activation of growth factor receptor mutations or overproduction of growth factor in an autocrine manner (65). Therefore, the other mechanism of Eps8 involvement in cell proliferation will be discussed from these two angles.

The most typical example of growth factor receptor activation is the increased expression or activity of various RTKs. Growth factors, such as EGF, bind to RTKs; stimulate intrinsic protein-tyrosine kinase activity in cells; and autophosphorylate several tyrosine residues in the cytoplasmic domain of RTKs. Multiple signal transduction cascades are initiated, particularly the RAS/MAPK pathway, which induces cell proliferation $(66,67)$.

RAS/MAPK signalling affects gene transcription by regulating the activity of transcription factors encoded by direct early genes and FOXM1, a forkhead box transcription factor (68). FOXM1 is a key regulator of the cell cycle process factor (69) and plays a pivotal role in cell proliferation (70). Notably, FOXM1 and Eps8 are activated by mitosis signals and upregulated in cancer. Eps 8 enhances the activity of the FOXM1 promoter. First, Eps8 is a protein associated with FOXM1 that stimulates FOXM1 to upregulate the expression of CXCL5, thereby increasing cell proliferation in a PI3K/AKT-dependent manner $(43,71)$. It is worth mentioning that Eps8 contains a hypothetical nuclear localization signal (NLS), which colocalizes with FOXM1 in the G2/M phase (2). The inhibition of CRM1/exportin1-mediated nuclear output enhanced the nuclear translocation of Eps8. In addition, Eps8 depletion inhibited the expression of FOXM1 and the FOXM1 target CCNB1 and slowed the G2/M transition in cervical cancer cells (72). In conclusion, these findings support the novel nuclear partnering role of Eps8 with FOXM1 in regulating cell proliferation.

Eps8 is a downstream component of EGF and stimulates growth factors (2). Eps8 protein levels and downstream phosphorylated ERK are upregulated in human pituitary tumours, 
and Eps8 cells proliferate more strongly under conditions full of growth factors and growth restriction (16). Further studies demonstrated that epidermal growth factor activated robust amplification of ERK and moderate upregulation of AKT in Eps8-overexpressing cells (16). Moreover, the inhibition or silencing of Eps8 by MAPK kinase could weaken the proliferation of cells stimulated by growth factor; in addition, blocking the PI3K pathway or silencing Eps8 could result in the loss of protection of Eps8 against apoptosis induced by growth factor depletion, subsequently decreasing cell apoptosis. Consequently, the increased expression of Eps8 results in an overreaction of the cells to the activation of local growth factors. Accordingly, the MAPK pathway is activated through ERK, and the PI3K pathway is activated through AKT, finally triggering cell proliferation and antiapoptotic responses (16).

FoxO3a/PI3K/AKT signalling pathway and tumour proliferation. It is generally considered that Eps8 facilitates the EGFR-induced PI3K/AKT pathway and achieves tumour growth at least in part by inhibiting FoxO3a $(73,74)$. FoxO3a is a well-known downstream transcription factor of the PI3K/AKT pathway (74) and is essential for differentiation (75). Recent studies on NSCLC have revealed that the Eps8 expression level is greatly increased in both cells and tissues (21). The mechanism is that Eps8 expression is negatively correlated with FoxO3a. FoxO3a inhibits the level of Eps8 by directly binding to the Eps8 gene promoter and forms a negative cycle in the EGFR pathway (21). Apart from non-small cell lung cancer, glioblastoma and breast cancer have also been revealed to downregulate Eps8 to inhibit proliferation both in vivo and in vitro $(20,22)$. Notably, the silencing of Eps8 in pancreatic ductal adenocarcinoma (PDAC) and oral squamous cells did not affect cell proliferation but inhibited other biological functions $(15,19)$. Herein, we emphasize that the aberrant expression of genes in tumours may affect some or all biological functions.

\section{Role and mechanisms of EPS8 in tumour invasion and} metastasis

Eps8 regulates tumour cell invasion and metastasis. Cell migration is a complex process involving reorganization of the actin cytoskeleton (76-78). Rho GTPase along with other cell processes regulates the organization of the actin cytoskeleton to promote coordinated changes in cell behaviour, in which members of Rho GTPases, including Rac, Cdc42 and Rho, affect different aspects of tumour cell motility (76-78). Rac promotes the formation of actin-rich membrane ruffle at the leading edge of migrating cells, called Lamellipodia (77). Cdc42 modulates cell polarity and the formation of filopodia, thus controlling the direction of cell movement, and Rho facilitates stress fibre formation and maintains focal adhesion at the rear of the cells (78). In addition, Ras has also been revealed to participate in cell motility and function downstream of $\mathrm{G}_{\mathrm{i}}$ to mediate ovarian cancer cell migration (79). Ras can activate Rac through Tiam1 (80), b-PIX (81), or the SOS1/Eps8/Abi1 tricomplex $(32,82)$. Notably, av $\beta 6$ and a5b1 integrin-dependent activation of Racl is mediated by Eps8. The downregulation of Eps8 or Rac1 inhibits integrin-dependent cell migration, whereas the transient expression of active Rac1 restores migration in cells with suppressed Eps8 expression (83).
Coactivation of Eps8 with F-actin has been revealed to occur primarily in pancreatic cancer cells. Eps8 is involved in cell morphology and protein skeleton, which determines cell migration. Moreover, Eps8 was located at the tips of F-actin filaments, filopodia, and the leading edge of cells. Eps8 knockdown altered cell shape and actinomycin-based cytoskeletal structures and impaired the formation of protuberance and intercellular connections (13).

The earliest studies reported that Eps8 overexpression encodes cell growth in fibroblasts (7), and its potential role in the development of human cancer has gradually been confirmed. Eps8 mRNA expression levels are sequentially increased in primary tumours, metastases, and malignant ascites, and Eps8 mRNA is predominantly expressed in advanced colorectal cancer (84). Similarly, Eps8 overexpression is highly associated with lymph node metastasis and parametrium invasion of cervical cancer (14). Conversely, silencing Eps8 expression blocks migration and invasion of human glioblastoma cell lines (85). In conclusion, Eps8 serves as a signalling intermediate for tumour invasion and metastasis.

SOS1/EPS8/Abil tricomplex signalling pathway and tumour invasion and metastasis. Lysophosphatidic acid (LPA), a growth factor-like phospholipid produced by ovarian cancer cells and secreted into the abdominal cavity, is uniquely associated with ovarian malignancies (86-89). High levels of LPA have been revealed in the ascites of ovarian cancer patients (87), which can effectively drive cell migration $(90,91)$. The process is accomplished through a signalling pathway consisting of the Ras-SOS1/Eps8/Abi1 tricomplex that stimulates Rac activation and cytoskeletal recombination, and LPA-induced Rac activation is a prerequisite for ovarian cancer metastasis. The integrity of SOS1/Eps8/Abi1 tricomplex may determine the possibility of ovarian cancer metastasis because silencing any member of the SOS1/Eps8/Abi1 tricomplex is not sufficient to reduce ovarian cancer cell migration and metastatic colonization. The three members of the complex play their roles and are interrelated. SOS1 serves as a Rac-specific guanine nucleotide exchange factor (GEF) and ultimately induces Rac-regulated cytoskeletal recombination and cell migration (92-94). Eps8 acts as a substrate for tyrosine kinase receptors. Abi1 is a scaffold protein that connects SOS1 and Eps8 $(73,95)$. Abi1 binds with SOS1 through its SH3 domain (96) and subsequently binds to the SH3 domain of Eps8.

Currently, studies have confirmed that the use of inhibitory peptides can disrupt specific protein-protein interactions and related biological behaviour (97-99). As small molecules, inhibitory peptides exhibit significant potential for clinical application (100). Moreover, short peptides have been successfully used to interfere with signalling pathways as a new cancer treatment $(101,102)$. Based on this activity, researchers selected Abil as the target for the design of short inhibitory peptides and successfully developed peptides capable of inhibiting the interaction between Eps8-Abi1 and ABI1-SOS1 to prevent the formation of the SOS1/Eps8/Abil tricomplex, thereby suppressing the invasion and metastasis of ovarian cancer (103). Biomedicine has made notable progress in tumour treatment, offering more opportunities to identify disease treatment targets $(102,103)$. 
EPS8/IRSp53 complexes and tumour invasion and metastasis. IRSp53, a protein crucial in cell mobilization, not only acts as a physical link between Rho GTPases and actin dynamics (104) but also serves as one of the Eps8 adapters from the human brain cDNA library. First, the interplay between Eps8 and IRSp53 increases Rac activation and cell migration in human fibrosarcoma cells (105). Additionally, this interaction contributes to Src-mediated transformation. Through Src activation, EGF increases the formation of the Eps8/IRSp53 complex in HeLa cells; the activation of AKT, ERK and Stat3; and the enhancement of cyclin D1 (106). Furthermore, the Eps8-IRSp53 complex collaboratively activates Rac by enhancing the formation of the Eps8-Abi-1-SOS-1 complex in fibroblasts and various cancer cell lines (36).

ERK/MMP9/P53 signalling pathway and tumour invasion and metastasis. In addition to affecting cell proliferation, Eps8 modulates the ERK signalling cascade and upregulates MMPs (such as MMP-9) and other matrix metalloproteinases to promote tumour cell invasion, leading to extracellular matrix remodelling (20,107). MMP-9 principally functions in EGF- and SF/HGF-induced migration, and attenuation of MMP-9 activity impairs receptor tyrosine kinase-dependent SCC mobility (108). Research has revealed that Eps8 overexpression induces cell migration and invasion in vitro and tumorigenicity in vivo, which depends on the activity of MMP-9 (107). Notably, Eps8 knockdown had no effect on ERK but reduced the levels of phosphorylated ERK and MMP9 while enhancing p53. Subsequently, the expression levels of the mitotic target genes c-Myc and cyclin D1, which are downstream of ERK signalling, were downregulated and finally inhibited the migration of breast cancer cells. Furthermore, attenuation of Eps8 suppressed part of the EMT-like transformation, significantly increased E-cadherin, and diminished $\mathrm{N}$-cadherin and vimentin. Notably, the number and size of EGF-induced Eps8-provoked filamentous pseudopods were also decreased (20). In short, Eps8 has been revealed to regulate breast cancer cell migration and invasion at least in part by affecting ERK signalling, MMP9, p53 and EMT markers.

Other factors target EPS8 to regulate tumour invasion and metastasis. MicroRNAs (miRNAs) are a group of 14-25 bp noncoding RNA (ncRNA) molecules (109) that regulate gene expression by inhibiting translation or cutting mRNA in a sequence-dependent manner (110) and are important regulators in the process of tumour metastasis (111). Studies have provided evidence for their roles in numerous types of tumours $(112,113)$. Initially, miR-345 was revealed to prevent GC cell metastasis by inhibiting the epithelial-mesenchymal transition (EMT) (114). Subsequently, Zhang et al revealed that Eps8 was a downstream target of miR-345 and that miR-345 inhibited GC cell migration, EMT and the CSC phenotype by inactivating the Rac1 signalling pathway (115). In addition, antitumour miR-130b-5p and numerous downstream genes mediated by Eps8 were closely involved in the aggressiveness of PDAC (23).

In addition to the pathways aforementioned, Eps8 also mediates tumour cell metastasis through other approaches. FOXM1 overexpression not only promotes cell proliferation but also increases cell migration $(71,116)$, and targeted inhibition of CXCL5 or AKT reduces Eps8-expressing cell migration (71). Therefore, Eps8 and FOXM1 may mediate cell migration through a series of common downstream components. In addition, the well-known PI3K/AKT cascade is activated by the binding of EGF to its receptor to increase migration and invasion in cancers (117). In particular, FoxO3a is also an important downstream target of the PI3K/AKT pathway (118) that inhibits the expression of Eps8 to prevent the migration and invasion of non-small cell lung cancer. The phosphorylation and translocation of FoxO3a are caused by EGF (118). Additionally, Eps8-induced FAK is overexpressed in numerous tumours and is strongly correlated with tumour aggressiveness (57,58). Maa et al demonstrated that Eps8 and FAK form a complex in regulating cell migration; however, the underlying mechanism remains unknown (12).

EPS8regulates tumour resistance, prognosis and angiogenesis. Eps8 is clearly associated with cellular responses to cisplatin, paclitaxel and imatinib. and cancer cells were more sensitive to drug therapy after Eps8 knockout $(14,25,119)$. Notably, Gorsic et al developed the Eps8 inhibitor miramycin A as a potential drug to improve the treatment index of cisplatin, which decreased the expression of Eps8, resulting in an augmentation in cell sensitivity to cisplatin that was significantly more pronounced in tumour cell lines than in lymphoblastoid cell lines (LCLs) (119). Mechanistically, on one hand, Eps8 increases p53 and decreases Src and AKT in such a way that HeLa and SiHa cells are sensitive to chemotherapeutic drugs (14). On the other hand, PI3K/AKT is an important cascade reaction of tumour chemotherapy resistance (120), and EGFR is the key to activating the PI3K/AKT signalling pathway (121). In general, EGFR TKI resistance is divided into 'on target' and 'off-target'. 'On-target' signifies that drug resistance is mainly caused by the variation of original drug targets, and 'off-target' refers to the activation of parallel signalling pathways (122). PI3K/AKT plays a regulatory role in both on-target and off-target resistance. PI3K/AKT is highly activated in human cancer and is used as a therapeutic target (123-126). As a downstream molecule of PI3K/AKT, FoxO3a (75) inhibits the expression of the Eps8 signalling protein by directly binding to the promoter of the Eps8 gene. The signalling pathways inhibited and promoted by Eps 8 are connected together to form a negative regulatory loop, which bypasses EGFR and reduces the activity of the PI3K/AKT pathway. Thus, it may affect the EGFR-TKI resistance in both 'on target' and 'off-target'. Therefore, FoxO3a may represent a core of the EGFR TKI resistance signalling network. EGFR and FoxO3a negatively regulate each other in the growth factor signalling network to maintain the physiological and biochemical functions of cells (21).

Eps8 expression in cancer cells may be a crucial biomarker of the prognosis of patients, which is closely concerned with the survival of patients and has clinical significance. Generally, the higher the expression of Eps8 in the early stage, the lower the survival rate. The overall survival (OS) of patients with expression of Eps8 was significantly lower than that of patients not expressing Eps8. Thus, Eps8 is considered an independent predictor of poor OS (19).

Tumours need to absorb nutrients from blood vessels to grow (127), therefore, controlling tumour-related angiogenesis 
is an attractive strategy to limit tumour progression. Li et al confirmed that FOXM1b has a direct and significant relationship with the transactivation of vascular endothelial growth factor expression and increased angiogenesis (116). In view of the fact that Eps8, as an adapter protein of FOXM1, mediates tumorigenesis and development, we hypothesise that Eps8 may also mediate tumour angiogenesis. Regrettably, the relationship between Eps8 and tumour angiogenesis remains unknown but is worthy of further exploration.

EPS8 regulates tumour immunotherapy. Immunotherapy is a popular method for cancer therapy and has achieved outstanding clinical effects. There is evidence that the host immune response can affect the survival of patients (128). Tumour-associated antigen (TAA) can produce specific cytotoxic T lymphocytes (CTLs). The recognition and identification of TAAs are important for the development of cancer immunotherapy (129). Eps8 is involved in the regulation of tumour progression and may be an ideal antigen because the new HLA-A*242-restricted epitope from Eps8 can be used as a new peptide inhibitor to inhibit the Eps8/EGFR interaction (130). In addition, studies have revealed that Eps8 protein increases the secretion of interleukin (IL)-12 into the culture supernatant of dendritic cells (DCs) and induces a significant CTL response, T-cell proliferation and high levels of interferon (IFN)- $\gamma$ (131). Given the relationship between Eps8 and tumour immunity, it may provide potential immunosuppressants for tumour treatment and create new diagnostic and treatment methods for clinicians, thus benefiting an increasing number of tumour patients.

\section{Role and molecular mechanism of Eps8 in haematological tumours}

Eps8 extensively functions as an oncogene in a wide range of solid tumours (10-23). In recent years, growing evidence has indicated that Eps8 is important for the proliferation, apoptosis and prognosis of haematological tumours, demonstrating the potential of Eps8-targeted therapy for leukaemia (24-27). Wang et al detected the expression of Eps8 mRNA and protein in 6 types of malignant haematological tumour cells (132). The results indicated that Eps8 mRNA and protein levels are not completely consistent. This study demonstrated the aberrant expression of Eps8 in malignant haematological tumours for the first time, providing a preliminary theoretical basis for the screening of new targets for the treatment of malignant haematological tumours.

Huang et al knocked out Eps8 in CML and MM cells, resulting in decreased proliferation and increased apoptosis. In addition, the absence of Eps 8 inhibited cell survival, migration and invasion, and induced drug sensitivity $(25,27)$. Eps8 was revealed to regulate the proliferation, apoptosis and chemosensitivity of BCR-ABL-positive cells by mediating the BCR-ABL/PI3K/AKT/mTOR pathway (25). Therefore, Eps8-targeted inhibitors alone or in combination with tyrosine kinase inhibitors may represent an exclusive strategy for refractory and drug-resistant CML patients. Notably, mithramycin (MTM), a specific Eps8 inhibitor, exhibited anti-MM activity in xenograft tumour models and suppressed the expression of Eps8 and related pathways (27). Sun et al hypothesized that trichostatin A (TSA), a panhistone deacetylase inhibitor (HDACi), could attenuate Eps8 and its downstream phosphorylated ERK1/2 pathway, thereby reducing the survival rate of Burkitt's lymphoma (BL) cells and inducing apoptosis and cell arrest at $\mathrm{G}_{0} / \mathrm{G}_{1}(133)$.

To further improve the prognosis of cancer patients, researchers analysed ALL and AML and revealed that high expression of the Eps8 gene predicted poor prognosis (132). As aforementioned Eps8 contains nuclear localization signals, and the release of Eps8 via tyrosine kinases creates a nuclear targeting signal responsible for the intracellular molecular mechanism of nuclear translocation (26). Castagnino et al observed that part of Eps8 was indeed translocated to the nucleus, upregulating the expression of Eps8 (41). Notably, the synthetic cell-penetrating peptide (CP-Eps8-NLS) derived from the nuclear localization signal of Eps8 could pass through the cell membrane and specifically interfere with the nuclear transport of Eps8. CP-Eps8-NLS exhibited anti-AML activity in various AML cell types and a synergistic effect with chemotherapeutic drugs in vivo and in vitro. CP-Eps8-NLS has been revealed to downregulate the expression of Eps8, PI3K/AKT and MAPK/ERK-related pathway targets; promote apoptosis and cell cycle arrest; and inhibit proliferation and cell viability (26). In particular, the NLS of Eps8 may represent a new target for further inhibitor design to interfere with Eps8-dependent AML progression.

In summary, Eps8 is likely to become a target for monitoring and treating haematological malignant tumours and exhibits markedly broad research and application prospects. Research on Eps8 and malignant haematological tumours has just started, and related basic and clinical research is markedly limited. Thus, more knowledge is required.

\section{Conclusion and future perspective}

The oncogene Eps8 is unusually expressed in solid tumours and haematological malignant tumours and represents an intriguing tumour biomarker linked to cellular signalling pathways. Recent evidence has indicated the importance of Eps8 in tumorigenesis, proliferation, migration, metastasis, drug resistance and poor prognosis in cancer patients. However, more tumour models and a large number of clinical trials are still required to verify its effect and clarify the broader mechanisms, and in the future, every effort will be made to find new directions for cancer treatment. Collectively, Eps8 is closely related to the occurrence and development of malignant tumours, and the potential of Eps8 in targeted applications and cancer drug development may be expanded with further research.

\section{Acknowledgements}

Not applicable.

\section{Funding}

The review was funded by the National Natural Science Foundation of China (grant no. 81860463), the Science and Technology Department of Yunnan Province and Kunming Medical University Project Foundation 
[grant no. 2018FE001(-003)], the Science and Technology Department of Yunnan Province and Kunming Medical University Project Foundation [grant no. 2017FE468(-067)].

\section{Availability of data and materials}

Not applicable.

\section{Authors' contributions}

CQ and ML designed the study and revised the manuscript. KL and LZ performed research and drafted the manuscript, analyzed data and wrote the final version of the manuscript. YL, HZ and HY participated in the conception of the study. All authors read and approved the final manuscript.

\section{Ethics approval and consent to participate}

Not applicable.

\section{Patient consent for publication}

Not applicable.

\section{Competing interests}

The authors declare that they have no competing interests.

\section{References}

1. Correction: Mitochondrial sirtuins in cancer: Emerging roles and therapeutic potential. Cancer Res 76: 3655, 2016.

2. Fazioli F, Minichiello L, Matoska V, Castagnino P, Miki T, Wong WT and Di Fiore PP: Eps8, a substrate for the epidermal growth factor receptor kinase, enhances EGF-dependent mitogenic signals. EMBO J 12: 3799-3808, 1993.

3. Wong WT, Carlomagno F, Druck T, Barletta C, Croce CM, Huebner K, Kraus MH and Di Fiore PP: Evolutionary conservation of the EPS8 gene and its mapping to human chromosome 12q23-q24. Oncogene 9: 3057-3061, 1994.

4. Tocchetti A, Confalonieri S, Scita G, Di Fiore PP and Betsholtz C: In silico analysis of the EPS8 gene family: Genomic organization, expression profile, and protein structure. Genomics 81 : 234-244, 2003.

5. Di Fiore PP and Scita G: Eps8 in the midst of GTPases. Int J Biochem Cell Biol 34: 1178-1183, 2002.

6. Offenhäuser N, Borgonovo A, Disanza A, Romano P, Ponzanelli I, Iannolo G, Di Fiore PP and Scita G: The eps8 family of proteins links growth factor stimulation to actin reorganization generating functional redundancy in the Ras/Rac pathway. Mol Biol Cell 15: 91-98, 2004.

7. Maa MC, Hsieh CY and Leu TH: Overexpression of p97Eps8 leads to cellular transformation: Implication of pleckstrin homology domain in p97Eps8-mediated ERK activation. Oncogene 20: 106-112, 2001.

8. Avantaggiato V, Torino A, Wong WT, Di Fiore PP and Simeone A: Expression of the receptor tyrosine kinase substrate genes eps8 and eps15 during mouse development. Oncogene 11: 1191-1198, 1995.

9. Ion A, Crosby AH, Kremer H, Kenmochi N, Van Reen M, Fenske C, Van Der Burgt I, Brunner HG, Montgomery K, Kucherlapati RS, et al: Detailed mapping, mutation analysis, and intragenic polymorphism identification in candidate Noonan syndrome genes MYL2, DCN, EPS8, and RPL6. J Med Genet 37: 884-886, 2000.

10. Huang Y, Prasad M, Lemon WJ, Hampel H, Wright FA, Kornacker K, LiVolsi V, Frankel W, Kloos RT, Eng C, et al: Gene expression in papillary thyroid carcinoma reveals highly consistent profiles. Proc Natl Acad Sci USA 98: 15044-15049, 2001.
11. Wang W, Wyckoff JB, Frohlich VC, Oleynikov Y, Hüttelmaier S, Zavadil J, Cermak L, Bottinger EP, Singer RH, White JG, et al: Single cell behavior in metastatic primary mammary tumors correlated with gene expression patterns revealed by molecular profiling. Cancer Res 62: 6278-6288, 2002.

12. Maa MC, Lee JC, Chen YJ, Chen YJ, Lee YC, Wang ST, Huang CC, Chow NH and Leu TH: Eps8 facilitates cellular growth and motility of colon cancer cells by increasing the expression and activity of focal adhesion kinase. J Biol Chem 282: 19399-19409, 2007.

13. Welsch T, Endlich K, Giese T, Büchler MW and Schmidt J: Eps8 is increased in pancreatic cancer and required for dynamic actin-based cell protrusions and intercellular cytoskeletal organization. Cancer Lett 255: 205-218, 2007.

14. Chen YJ, Shen MR, Chen YJ, Maa MC and Leu TH: Eps8 decreases chemosensitivity and affects survival of cervical cancer patients. Mol Cancer Ther 7: 1376-1385, 2008.

15. Yap LF, Jenei V, Robinson CM, Moutasim K, Benn TM, Threadgold SP, Lopes V, Wei W, Thomas GJ and Paterson IC: Upregulation of Eps8 in oral squamous cell carcinoma promotes cell migration and invasion through integrin-dependent Rac1 activation. Oncogene 28: 2524-2534, 2009.

16. Xu M, Shorts-Cary L, Knox AJ, Kleinsmidt-DeMasters B, Lillehei $\mathrm{K}$ and Wierman ME: Epidermal growth factor receptor pathway substrate 8 is overexpressed in human pituitary tumors: Role in proliferation and survival. Endocrinology 150: 2064-2671, 2009.

17. Bashir M, Kirmani D, Bhat HF, Baba RA, Hamza R, Naqash S, Wani NA, Andrabi KI, Zargar MA and Khanday FA: P66shc and its downstream Eps8 and Rac1 proteins are upregulated in esophageal cancers. Cell Commun Signal 8: 13, 2010.

18. Chen H, Wu X, Pan ZK and Huang S: Integrity of SOS1/EPS8/ABI1 tri-complex determines ovarian cancer metastasis. Cancer Res 70: 9979-9990, 2010.

19. Chu PY, Liou JH, Lin YM, Chen CJ, Chen MK, Lin SH, Yeh CM, Wang HK, Maa MC, Leu TH, et al: Expression of Eps8 correlates with poor survival in oral squamous cell carcinoma. Asia Pac J Clin Oncol 8: e77-e81, 2012.

20. Chen C, Liang Z, Huang W, Li X, Zhou F, Hu X, Han M, Ding X and Xiang S: Eps8 regulates cellular proliferation and migration of breast cancer. Int J Oncol 46: 205-214, 2015.

21. Wen Q, Jiao X, Kuang F, Hou B, Zhu Y, Guo W, Sun G, Ba Y, Yu D, Wang D, et al: FoxO3a inhibiting expression of EPS8 to prevent progression of NSCLC: A new negative loop of EGFR signaling. EBioMedicine 40: 198-209, 2019.

22. Yang G, Lu YB and Guan QL: EPS8 is a potential oncogene in glioblastoma. Onco Targets Ther 12: 10523-10534, 2019.

23. Fukuhisa H, Seki N, Idichi T, Kurahara H, Yamada Y, Toda H, Kita Y, Kawasaki Y, Tanoue K, Mataki Y, et al: Gene regulation by antitumor miR-130b-5p in pancreatic ductal adenocarcinoma: The clinical significance of oncogenic EPS8. J Hum Genet 64: 521-534, 2019.

24. He YZ, Liang Z, Wu MR, Wen Q, Deng L, Song CY, Wu BY, Tu SF, Huang R and Li YH: Overexpression of EPS8 is associated with poor prognosis in patients with acute lymphoblastic leukemia. Leuk Res 39: 575-581, 2015.

25. Huang R, Liu H, Chen Y, He Y, Kang Q, Tu S, He Y, Zhou X, Wang L, Yang J, et al: EPS8 regulates proliferation, apoptosis and chemosensitivity in BCR-ABL positive cells via the BCR-ABL/PI3K/AKT/mTOR pathway. Oncol Rep 39: 119-128, 2018.

26. Chen Y, Xie X, Wu A, Wang L, Hu Y, Zhang H and Li Y: A synthetic cell-penetrating peptide derived from nuclear localization signal of EPS8 exerts anticancer activity against acute myeloid leukemia. J Exp Clin Cancer Res 37: 12, 2018.

27. Zhang H, Zhou L, Zhou W, Xie X, Wu M, Chen Y, Hu Y, Du J, $\mathrm{He}$ Y and Li Y: EPS8-mediated regulation of multiple myeloma cell growth and survival. Am J Cancer Res 9: 1622-1634, 2019.

28. Behlouli A, Bonnet C, Abdi S, Bouaita A, Lelli A, Hardelin JP, Schietroma C, Rous Y, Louha M, Cheknane A, et al: EPS8, encoding an actin-binding protein of cochlear hair cell stereocilia, is a new causal gene for autosomal recessive profound deafness. Orphanet J Rare Dis 9: 55, 2014.

29. Morton CJ, Pugh DJ, Brown EL, Kahmann JD, Renzoni DA and Campbell ID: Solution structure and peptide binding of the SH3 domain from human Fyn. Structure 4: 705-714, 1996.

30. Matoskova B, Wong WT, Salcini AE, Pelicci PG and Di Fiore PP: Constitutive phosphorylation of eps8 in tumor cell lines: Relevance to malignant transformation. Mol Cell Biol 15: 3805-3812, 1995. 
31. Shi X, Betzi S, Lugari A, Opi S, Restouin A, Parrot I, Martinez J, Zimmermann $\mathrm{P}$, Lecine $\mathrm{P}$, Huang $\mathrm{M}$, et al: Structural recognition mechanisms between human Src homology domain $3(\mathrm{SH} 3)$ and ALG-2-interacting protein X (Alix). FEBS Lett 586: 1759-1764, 2012.

32. Scita G, Nordstrom J, Carbone R, Tenca P, Giardina G, Gutkind S, Bjarnegård M, Betsholtz C and Di Fiore PP: EPS8 and E3B1 transduce signals from Ras to Rac. Nature 401: 290-293, 1999.

33. Lanzetti L, Rybin V, Malabarba MG, Christoforidis S, Scita G, Zerial M and Di Fiore PP: The Eps8 protein coordinates EGF receptor signalling through Rac and trafficking through Rab5. Nature 408: 374-377, 2000.

34. Kishan KV, Newcomer ME, Rhodes TH and Guilliot SD: Effect of $\mathrm{pH}$ and salt bridges on structural assembly: Molecular structures of the monomer and intertwined dimer of the Eps8 SH3 domain. Protein Sci 10: 1046-1055, 2001.

35. Inobe M, Ki K, Miyagoe Y, Yi N and Takeda S: Identification of EPS8 as a Dvl1-associated molecule. Biochem Biophys Res Commun 266: 216-221, 1999.

36. Funato Y, Terabayashi T, Suenaga N, Seiki M, Takenawa T and Miki H: IRSp53/Eps8 complex is important for positive regulation of Rac and cancer cell motility/invasiveness. Cancer Res 64: 5237-5244, 2004.

37. Disanza A, Mantoani S, Hertzog M, Gerboth S, Frittoli E, Steffen A, Berhoerster K, Kreienkamp HJ, Milanesi F, Di Fiore PP, et al: Regulation of cell shape by Cdc42 is mediated by the synergic actin-bundling activity of the Eps8-IRSp53 complex. Nat Cell Biol 8: 1337-1347, 2006.

38. Prieto-Echagüe V, Chan PM, Craddock BP, Manser E and Miller WT: PTB domain-directed substrate targeting in a tyrosine kinase from the unicellular choanoflagellate Monosiga brevicollis. PLoS One 6: e19296, 2011.

39. Forman-Kay JD and Pawson T: Diversity in protein recognition by PTB domains. Curr Opin Struct Biol 9: 690-695, 1999.

40. Slupsky CM, Gentile LN, Donaldson LW, Mackereth CD Seidel JJ, Graves BJ and McIntosh LP: Structure of the Ets-1 pointed domain and mitogen-activated protein kinase phosphorylation site. Proc Natl Acad Sci USA 95: 12129-12134, 1998.

41. Castagnino P, Biesova Z, Wong WT, Fazioli F, Gill GN and Di Fiore PP: Direct binding of eps8 to the juxtamembrane domain of EGFR is phosphotyrosine- and SH2-independent. Oncogene 10: 723-729, 1995.

42. Disanza A, Carlier MF, Stradal TE, Didry D, Frittoli E, Confalonieri S, Croce A, Wehland J, Di Fiore PP and Scita G: Eps8 controls actin-based motility by capping the barbed ends of actin filaments. Nat Cell Biol 6: 1180-1188, 2004.

43. Scita G, Tenca P, Areces LB, Tocchetti A, Frittoli E, Giardina G, Ponzanelli I, Sini P, Innocenti M and Di Fiore PP: An effector region in Eps 8 is responsible for the activation of the Rac-specific GEF activity of Sos-1 and for the proper localization of the Rac-based actin-polymerizing machine. J Cell Biol 154: 1031-1044, 2001.

44. Kirkland G, Paizis K, Wu LL, Katerelos M and Power DA Heparin-binding EGF-like growth factor mRNA is upregulated in the peri-infarct region of the remnant kidney model: In vitro evidence suggests a regulatory role in myofibroblast transformation. J Am Soc Nephrol 9: 1464-1473, 1998.

45. Miao H, Wei BR, Peehl DM, Li Q, Alexandrou T, Schelling JR, Rhim JS, Sedor JR, Burnett E and Wang B: Activation of EphA receptor tyrosine kinase inhibits the Ras/MAPK pathway. Nat Cell Biol 3: 527-530, 2001.

46. Carpenter G and Cohen S: Epidermal growth factor. Annu Rev Biochem 48: 193-216, 1979.

47. Buday L and Downward J: Epidermal growth factor regulates p21ras through the formation of a complex of receptor, Grb2 adapter protein, and Sos nucleotide exchange factor. Cell 73 611-620, 1993

48. Ozanne B, Richards CS, Hendler F, Burns D and Gusterson B Over-expression of the EGF receptor is a hallmark of squamous cell carcinomas. J Pathol 149: 9-14, 1986.

49. Rubin Grandis J, Zeng Q and Drenning SD: Epidermal growth factor receptor-mediated stat3 signaling blocks apoptosis in head and neck cancer. Laryngoscope 110: 868-874, 2000.

50. Song JI and Grandis JR: STAT signaling in head and neck cancer. Oncogene 19: 2489-2895, 2000.

51. Grandis JR, Drenning SD, Chakraborty A, Zhou MY, Zeng Q, Pitt AS and Tweardy DJ: Requirement of Stat 3 but not Stat1 activation for epidermal growth factor receptor- mediated cell growth In vitro. J Clin Invest 102: 1385-1392, 1998.
52. Minden A, Lin A, McMahon M, Lange-Carter C, Dérijard B, Davis RJ, Johnson GL and Karin M: Differential activation of ERK and JNK mitogen-activated protein kinases by Raf-1 and MEKK. Science 266: 1719-1723, 1994.

53. Lin A, Minden A, Martinetto H, Claret FX, Lange-Carter C, Mercurio F, Johnson GL and Karin M: Identification of a dual specificity kinase that activates the Jun kinases and p38-Mpk2. Science 268: 286-290, 1995.

54. Minden A, Lin A, Claret FX, Abo A and Karin M: Selective activation of the JNK signaling cascade and c-Jun transcriptional activity by the small GTPases Rac and Cdc42Hs. Cell 81: 1147-1157, 1995.

55. Schaller MD, Borgman CA and Parsons JT: Autonomous expression of a noncatalytic domain of the focal adhesion-associated protein tyrosine kinase pp125FAK. Mol Cell Biol 13: 785-791, 1993.

56. Hanks SK, Calalb MB, Harper MC and Patel SK: Focal adhesion protein-tyrosine kinase phosphorylated in response to cell attachment to fibronectin. Proc Natl Acad Sci USA 89: 8487-8491, 1992.

57. Parsons JT: Focal adhesion kinase: The first ten years. J Cell Sci 116: 1409-1416, 2003.

58. Hanks SK, Ryzhova L, Shin NY and Brábek J: Focal adhesion kinase signaling activities and their implications in the control of cell survival and motility. Front Biosci 8: d982-d996, 2003.

59. Yu CL, Meyer DJ, Campbell GS, Larner AC, Carter-Su C, Schwartz J and Jove R: Enhanced DNA-binding activity of a Stat3-related protein in cells transformed by the Src oncoprotein. Science 269: 81-83, 1995.

60. Bromberg JF, Horvath CM, Besser D, Lathem WW and Darnell JE Jr: Stat3 activation is required for cellular transformation by v-src. Mol Cell Biol 18: 2553-2558, 1998.

61. Turkson J, Bowman T, Garcia R, Caldenhoven E, De Groot RP and Jove R: Stat 3 activation by Src induces specific gene regulation and is required for cell transformation. Mol Cell Biol 18: 2545-2552, 1998

62. Leu TH, Yeh HH, Huang CC, Chuang YC, Su SL and Maa MC: Participation of p97Eps8 in Src-mediated transformation. J Biol Chem 279: 9875-9881, 2004.

63. Maa MC, Lai JR, Lin RW and Leu TH: Enhancement of tyrosyl phosphorylation and protein expression of eps 8 by v-Src. Biochim Biophys Acta 1450: 341-351, 1999.

64. Sachdev S, Bu Y and Gelman IH: Paxillin-Y118 phosphorylation contributes to the control of Src-induced anchorage-independent growth by FAK and adhesion. BMC Cancer 9: 12, 2009.

65. Hanahan D and Weinberg RA: Hallmarks of cancer: The next generation. Cell 144: 646-674, 2011

66. Ma RY, Tong TH, Cheung AM, Tsang AC, Leung WY and Yao KM: Raf/MEK/MAPK signaling stimulates the nuclear translocation and transactivating activity of FOXM1c. J Cell Sci 118: 795-806, 2005.

67. Koo CY, Muir KW and Lam EW: FOXM1: From cancer initiation to progression and treatment. Biochim Biophys Acta 1819: 28-37, 2012

68. Laoukili J, Kooistra MR, Brás A, Kauw J, Kerkhoven RM, Morrison A, Clevers H and Medema RH: FoxM1 is required for execution of the mitotic programme and chromosome stability. Nat Cell Biol 7: 126-136, 2005.

69. Costa RH: FoxM1 dances with mitosis. Nat Cell Biol 7: 108-110, 2005.

70. Kwok CT, Leung MH, Qin J, Qin Y, Wang J, Lee YL and Yao KM: The Forkhead box transcription factor FOXM1 is required for the maintenance of cell proliferation and protection against oxidative stress in human embryonic stem cells. Stem Cell Res 16: 651-661, 2016.

71. Wang H, The MT, Ji Y, Patel V, Firouzabadian S, Patel AA, Gutkind JS and Yeudall WA: EPS8 upregulates FOXM1 expression, enhancing cell growth and motility. Carcinogenesis 31: 1132-1141, 2010.

72. Ngan AWL, Grace Tsui M, So DHF, Leung WY, Chan DW and Yao KM: Novel nuclear partnering role of EPS8 with FOXM1 in regulating cell proliferation. Front Oncol 9: 154, 2019.

73. Innocenti M, Frittoli E, Ponzanelli I, Falck JR, Brachmann SM, Di Fiore PP and Scita G: Phosphoinositide 3-kinase activates Rac by entering in a complex with Eps8, Abi1, and Sos-1. J Cell Biol 160: 17-23, 2003

74. Chiu CF, Chang YW, Kuo KT, Shen YS, Liu CY, Yu YH, Cheng CC, Lee KY, Chen FC, Hsu MK, et al: NF- $\mathrm{BB}$-driven suppression of FOXO3a contributes to EGFR mutation-independent gefitinib resistance. Proc Natl Acad Sci USA 113: E2526-E2535, 2016. 
75. Accili D and Arden KC: FoxOs at the crossroads of cellular metabolism, differentiation, and transformation. Cell 117: 421-426, 2004.

76. Hall A: Rho GTPases and the actin cytoskeleton. Science 279: 509-514, 1998.

77. Nobes CD and Hall A: Rho, rac, and cdc42 GTPases regulate the assembly of multimolecular focal complexes associated with actin stress fibers, lamellipodia, and filopodia. Cell 81: 53-62, 1995.

78. Hall A and Nobes CD: Rho GTPases: Molecular switches that control the organization and dynamics of the actin cytoskeleton. Philos Trans R Soc Lond B Biol Sci 355: 965-970, 2000.

79. Bian D, Su S, Mahanivong C, Cheng RK, Han Q, Pan ZK, Sun P and Huang S: Lysophosphatidic acid stimulates ovarian cancer cell migration via a ras-MEK kinase 1 pathway. Cancer Res 64: 4209-4217, 2004

80. Lambert JM, Lambert QT, Reuther GW, Malliri A Siderovski DP, Sondek J, Collard JG and Der CJ: Tiam1 mediates Ras activation of Rac by a PI(3)K-independent mechanism Nat Cell Biol 4: 621-625, 2002.

81. Shin EY, Shin KS, Lee CS, Woo KN, Quan SH, Soung NK Kim YG, Cha CI, Kim SR, Park D, et al: Phosphorylation of $\mathrm{p} 85$ beta PIX, a Rac/Cdc42-specific guanine nucleotide exchange factor, via the Ras/ERK/PAK2 pathway is required for basic fibroblast growth factor-induced neurite outgrowth. J Biol Chem 277: 44417-44430, 2002.

82. Nimnual AS, Yatsula BA and Bar-Sagi D: Coupling of Ras and Rac guanosine triphosphatases through the Ras exchanger Sos. Science 279: 560-563, 1998

83. Tod J, Hanley CJ, Morgan MR, Rucka M, Mellows T, Lopez MA Kiely P, Moutasim KA, Frampton SJ, Sabnis D, et al: Pro-migratory and TGF- $\beta$-activating functions of $\alpha v \beta 6$ integrin in pancreatic cancer are differentially regulated via an Eps8-dependent GTPase switch. J Pathol 243: 37-50, 2017.

84. Nasri E, Wiesen LB, Knapik JA and Fredenburg KM: Eps8 expression is significantly lower in p16+ head and neck squamous cell carcinomas (HNSCCs) compared with p16- HNSCCs Hum Pathol 72: 45-51, 2018.

85. Cattaneo MG, Cappellini E and Vicentini LM: Silencing of Eps8 blocks migration and invasion in human glioblastoma cell lines. Exp Cell Res 318: 1901-1912, 2012.

86. Lengyel E: Ovarian cancer development and metastasis. Am J Pathol 177: 1053-1064, 2010.

87. Jesionowska A, Cecerska-Heryc E, Matoszka N and Dolegowska B: Lysophosphatidic acid signaling in ovarian cancer. J Recept Signal Transduct Res 35: 578-584, 2015.

88. Pua TL, Wang FQ and Fishman DA: Roles of LPA in ovarian cancer development and progression. Future Oncol 5: 1659-1673, 2009.

89. Fang X, Schummer M, Mao M, Yu S, Tabassam FH, Swaby R, Hasegawa Y, Tanyi JL, LaPushin R, Eder A, et al: Lysophosphatidic acid is a bioactive mediator in ovarian cancer Biochim Biophys Acta 1582: 257-264, 2002

90. Yu S, Murph MM, Lu Y, Liu S, Hall HS, Liu J, Stephens C, Fang X and Mills GB: Lysophosphatidic acid receptors determine tumorigenicity and aggressiveness of ovarian cancer cells J Natl Cancer Inst 100: 1630-1642, 2008.

91. Wang P, Wu X, Chen W, Liu J and Wang X: The lysophosphatidic acid (LPA) receptors their expression and significance in epithelial ovarian neoplasms. Gynecol Oncol 104: 714-720, 2007.

92.Pierre S, Bats AS and Coumoul X: Understanding SOS (Son of Sevenless). Biochem Pharmacol 82: 1049-1056, 2011.

93. Schmidt A and Hall A: Guanine nucleotide exchange factors for Rho GTPases: turning on the switch. Genes Dev 16: 1587-1609, 2002.

94. Innocenti M, Tenca P, Frittoli E, Faretta M, Tocchetti A, Di Fiore PP and Scita G: Mechanisms through which Sos-1 coordinates the activation of Ras and Rac. J Cell Biol 156 125-136, 2002.

95. Kotula L: Abi1, a critical molecule coordinating actin cytoskeleton reorganization with PI-3 kinase and growth signaling. FEBS Lett 586: 2790-2794, 2012.

96. Fan PD and Goff SP: Abl interactor 1 binds to sos and inhibits epidermal growth factor- and v-Abl-induced activation of extracellular signal-regulated kinases. Mol Cell Biol 20: 7591-7601, 2000.

97. Stone TA and Deber CM: Therapeutic design of peptide modulators of protein-protein interactions in membranes. Biochim Biophys Acta Biomembr 1859: 577-585, 2017.
98. Cunningham AD, Qvit N and Mochly-Rosen D: Peptides and peptidomimetics as regulators of protein-protein interactions. Curr Opin Struct Biol 44: 59-66, 2017.

99. Helmer D and Schmitz K: Peptides and peptide analogs to inhibit protein-protein interactions. Adv Exp Med Biol 917 $147-183,2016$

100. Fosgerau K and Hoffmann T: Peptide therapeutics: Current status and future directions. Drug Discov Today 20: 122-128, 2015.

101. Ellert-Miklaszewska A, Poleszak K and Kaminska B: Short peptides interfering with signaling pathways as new therapeutic tools for cancer treatment. Future Med Chem 9: 199-221, 2017.

102. Bae DG, Kim TD, Li G, Yoon WH and Chae CB: Anti-flt peptide, a vascular endothelial growth factor receptor 1-specific hexapeptide, inhibits tumor growth and metastasis. Clin Cancer Res 11: 2651-2561, 2005.

103. Yu X, Liang C, Zhang Y, Zhang W and Chen H: Inhibitory short peptides targeting EPS8/ABI1/SOS1 tri-complex suppress invasion and metastasis of ovarian cancer cells. BMC Cancer 19: $878,2019$.

104. Raftopoulou M and Hall A: Cell migration: Rho GTPases lead the way. Dev Biol 265: 23-32, 2004.

105. Miki H, Yamaguchi H, Suetsugu S and Takenawa T: IRSp53 is an essential intermediate between Rac and WAVE in the regulation of membrane ruffling. Nature 408: 732-735, 2000.

106. Liu PS, Jong TH, Maa MC and Leu TH: The interplay between Eps8 and IRSp53 contributes to Src-mediated transformation. Oncogene 29: 3977-3989, 2010.

107. Wang H, Patel V, Miyazaki H, Gutkind JS and Yeudall WA: Role for EPS8 in squamous carcinogenesis. Carcinogenesis 30 $165-174,2009$.

108. McCawley LJ, Li S, Wattenberg EV and Hudson LG: Sustained activation of the mitogen-activated protein kinase pathway. A mechanism underlying receptor tyrosine kinase specificity for matrix metalloproteinase-9 induction and cell migration. J Biol Chem 274: 4347-4353, 1999.

109. Ha M and Kim VN: Regulation of microRNA biogenesis. Nat Rev Mol Cell Biol 15: 509-524, 2014.

110. Liu WW, Meng J, Cui J and Luan YS: Characterization and Function of MicroRNA*s in Plants. Front Plant Sci 8: 2200 2017.

111. Wu WK, Lee CW, Cho CH, Fan D, Wu K, Yu J and Sung JJ: MicroRNA dysregulation in gastric cancer: A new player enters the game. Oncogene 29: 5761-5771, 2010.

112. Yu M, Xue H, Wang Y, Shen Q, Jiang Q, Zhang X, Li K, Jia M, Jia J, Xu J and Tian Y: miR-345 inhibits tumor metastasis and EMT by targeting IRF1-mediated mTOR/STAT3/AKT pathway in hepatocellular carcinoma. Int J Oncol 50: 975-983, 2017.

113. Ying X, Zhang W, Fang M, Zhang W, Wang C and Han L: miR-345-5p regulates proliferation, cell cycle, and apoptosis of acute myeloid leukemia cells by targeting AKT2. J Cell Biochem, 2018 (Epub ahead of print).

114. Feng A, Yuan X and Li X: MicroRNA-345 inhibits metastasis and epithelial-mesenchymal transition of gastric cancer by targeting FOXQ1. Oncol Rep 38: 2752-2760, 2017.

115. Zhang J, Wang C, Yan S, Yang Y, Zhang X and Guo W: miR-345 inhibits migration and stem-like cell phenotype in gastric cancer via inactivation of Rac1 by targeting EPS8. Acta Biochim Biophys Sin (Shanghai) 52: 259-267, 2020.

116. Li Q, Zhang N, Jia Z, Le X, Dai B, Wei D, Huang S, Tan D and $\mathrm{X}$ ie K: Critical role and regulation of transcription factor FoxM1 in human gastric cancer angiogenesis and progression. Cancer Res 69: 3501-3509, 2009

117. Kedmi M, Ben-Chetrit N, Körner C, Mancini M Ben-Moshe NB, Lauriola M, Lavi S, Biagioni F, Carvalho S, Cohen-Dvashi $\mathrm{H}$, et al: EGF induces microRNAs that target suppressors of cell migration: miR-15b targets MTSS1 in breast cancer. Sci Signal 8: ra29, 2015

118. Santo EE, Stroeken P, Sluis PV, Koster J, Versteeg R and Westerhout EM: FOXO3a is a major target of inactivation by PI3K/AKT signaling in aggressive neuroblastoma. Cancer Res 73: 2189-2198, 2013

119. Gorsic LK, Stark AL, Wheeler HE, Wong SS, Im HK and Dolan ME: EPS8 inhibition increases cisplatin sensitivity in lung cancer cells. PLoS One 8: e82220, 2013.

120. Smolensky D, Rathore K, Bourn J and Cekanova M: Inhibition of the PI3K/AKT Pathway Sensitizes Oral Squamous Cell Carcinoma Cells to Anthracycline-Based Chemotherapy In Vitro. J Cell Biochem 118: 2615-2624, 2017. 
121. Li F, Zhao X, Sun R, Ou J, Huang J, Yang N, Xu T, Li J, He X, Li C, et al: EGFR-rich extracellular vesicles derived from highly metastatic nasopharyngeal carcinoma cells accelerate tumour metastasis through PI3K/AKT pathway-suppressed ROS J Extracell Vesicles 10: e12003, 2020.

122. Rotow $\mathrm{J}$ and Bivona TG: Understanding and targeting resistance mechanisms in NSCLC. Nat Rev Cancer 17: 637-658, 2017.

123. Li H, Zeng J and Shen K: PI3K/AKT/mTOR signaling pathway as a therapeutic target for ovarian cancer. Arch Gynecol Obstet 290: 1067-1078, 2014.

124. Slomovitz BM and Coleman RL: The PI3K/AKT/mTOR pathway as a therapeutic target in endometrial cancer. Clin Cancer Res 18: 5856-5864, 2012.

125. Mabuchi S, Kuroda H, Takahashi R and Sasano T: The $\mathrm{PI} 3 \mathrm{~K} / \mathrm{AKT} / \mathrm{mTOR}$ pathway as a therapeutic target in ovarian cancer. Gynecol Oncol 137: 173-179, 2015.

126. Narayanankutty A: PI3K/Akt/ mTOR Pathway as a therapeutic target for colorectal cancer: A review of preclinical and clinical evidence. Curr Drug Targets 20: 1217-1226, 2019.

127. Jiang X, Wang J, Deng X, Xiong F, Zhang S, Gong Z, Li X, Cao K, Deng H, He Y, et al: The role of microenvironment in tumor angiogenesis. J Exp Clin Cancer Res 39: 204, 2020.

128. Kirkwood JM, Butterfield LH, Tarhini AA, Zarour H, Kalinski P and Ferrone S: Immunotherapy of cancer in 2012. CA Cancer J Clin 62: 309-335, 2012.
129. Novellino L, Castelli C and Parmiani G: A listing of human tumor antigens recognized by T cells: March 2004 update. Cancer Immunol Immunother 54: 187-207, 2005.

130.Xie X, Zhou W, Hu Y, Chen Y, Zhang H and Li Y: A dual-function epidermal growth factor receptor pathway substrate 8 (Eps8)-derived peptide exhibits a potent cytotoxic T lymphocyte-activating effect and a specific inhibitory activity. Cell Death Dis 9: 379, 2018 .

131. He YJ, Zhou J, Zhao TF, Hu LS, Gan JY, Deng L and Li Y: Eps8 vaccine exerts prophylactic antitumor effects in a murine model: A novel vaccine for breast carcinoma. Mol Med Rep 8: 662-668, 2013.

132. Wang L, Cai SH, Xiong WY, He YJ, Deng L and Li YH: Real-time quantitative polymerase chain reaction assay for detecting the eps8 gene in acute myeloid leukemia. Clin Lab 59: 1261-1269, 2013.

133. Sun P, Zhou X, He Y, Liu H, Wang Y, Chen Y, Li M, He Y, Li G and Li Y: Effect of trichostatin A on Burkitt's lymphoma cells: Inhibition of EPS8 activity through Phospho-Erk1/2 pathway. Biochem Biophys Res Commun 497: 990-996, 2018.

This work is licensed under a Creative Commons Attribution-NonCommercial-NoDerivatives 4.0 International (CC BY-NC-ND 4.0) License. 